\title{
Prognostic significance of tumor budding in rectal cancer biopsies before neoadjuvant therapy
}

\author{
Ailín C Rogers ${ }^{1,2}$, David Gibbons ${ }^{1,2}$, Ann M Hanly ${ }^{1,2}$, John MP Hyland ${ }^{1,2}$, P Ronan \\ O’Connell $^{1,2}$, Desmond C Winter ${ }^{1,2}$ and Kieran Sheahan ${ }^{1,2}$ \\ ${ }^{1}$ Centre for Colorectal Disease, St Vincent's University Hospital, Dublin, Ireland and ${ }^{2}$ School of Medicine and \\ Medical Science, University College Dublin, Dublin, Ireland
}

\begin{abstract}
Tumor budding is an increasingly important prognostic feature for pathologists to recognize. The aim of this study was to correlate intra-tumoral budding in pre-treatment rectal cancer biopsies with pathological response to neoadjuvant chemoradiotherapy and with long-term outcome. Data from a prospectively maintained database were acquired from patients with locally advanced rectal cancer who underwent neoadjuvant chemoradiotherapy. Pre-treatment rectal biopsies were retrospectively reviewed for evidence of intra-tumoral budding. Multivariate logistic regression was used to identify factors contributing to cancer-specific death, expressed as hazard ratios with $95 \%$ confidence intervals. Of the 185 patients with locally advanced rectal cancer, 89 patients met the eligibility criteria, of whom $18(20 \%)$ exhibited budding in a pre-treatment tumor biopsy. Intra-tumoral budding predicted a poor pathological response to neoadjuvant chemoradiotherapy (higher ypT stage, $P=0.032$; lymph node involvement, $P=0.018$; lymphovascular invasion, $P=0.004$; and

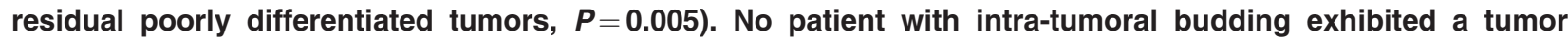
regression grade 1 or complete pathological response, providing a $100 \%$ specificity and positive predictive value for non-response to neoadjuvant chemoradiotherapy. Intra-tumoral budding was associated with a lower disease-free 5-year survival rate (33 vs $78 \%, P<0.001)$, cancer-specific 5-year survival rate $(61$ vs $87 \%, P=0.021)$ and predicted cancer-specific death (hazard ratio $3.51,95 \%$ confidence interval $1.03-11.93, P=0.040$ ). Intratumoral budding at diagnosis of rectal cancer identifies those who will poorly respond to neoadjuvant chemoradiotherapy and those with a poor prognosis.

Modern Pathology (2014) 27, 156-162; doi:10.1038/modpathol.2013.124; published online 26 July 2013
\end{abstract}

Keywords: biopsy; intra-tumoral budding; prognosis; rectal cancer

Tumor budding is a histological finding in epithelial cancers that occurs when tumor cells detach from the invasive tumor margin and migrate into the surrounding stroma. ${ }^{1,2}$ It likely represents dedifferentiation from epithelial cancer cells to tumor stem cells, which in turn may redifferentiate and invade locally or migrate to distant sites. $^{3,4}$ Tumor budding at the invasive tumor margin is an established negative prognostic factor in resected colorectal cancer. ${ }^{5-12}$ Widespread use of tumor budding as a prognostic factor has been impeded by a lack of standardized

Correspondence: Professor K Sheahan, MB, FRCPI, FRCPath, Centre for Colorectal Disease, St Vincent's University Hospital, Dublin 4, Ireland.

E-mail: K.Sheahan@st-vincents.ie

Received 26 January 2013; revised 31 May 2013; accepted 31 May 2013; published online 26 July 2013 histopathological criteria, highlighted recently by Mitrovic et al ${ }^{13}$ who state that 'although performing a tumor bud count is easy in theory, deciding what is or is not a bud can prove surprisingly difficult in practice'. Recent studies have addressed the role of tumor budding in resected colorectal specimens. ${ }^{14-16}$ Tumor budding within the entire tumor, rather than at the invasive front, is termed intra-tumoral budding ${ }^{17}$ and has been described in both colorectal cancer biopsy and resected specimens. ${ }^{18,19}$ This study addresses the prognostic and predictive power of tumor budding in biopsy specimens at the time of diagnosis of locally advanced rectal cancer.

Patients with the same stage of rectal cancer at initial diagnosis can have markedly different outcomes. ${ }^{20-22}$ Apart from TNM staging, reliable prognostic markers of rectal cancer are few and, apart from pre-operative carcinoembryonic antigen 
(CEA), ${ }^{23-25}$ not often used clinically. ${ }^{23}$ Tissue diagnosis usually depends on a superficial biopsy obtained at endoscopy from which adverse features such as tumor depth, lymphovascular invasion and perineural invasion cannot be determined. Current treatment options, including local excision, neoadjuvant therapy and radical surgical excision, are guided by relatively crude measurements of local and distant tumor extent and not by markers of tumor biology, which determine tumor response to treatment and ultimately patient survival. Therapeutic algorithms informed by markers of tumor biology are needed to ensure that maximal treatment, including neoadjuvant chemoradiotherapy, is given only when benefit is likely and, conversely, that local or minimally invasive alternatives are available when biologically appropriate.

The aim of this study was to investigate the potential value of intra-tumoral budding in pretreatment biopsies of rectal cancer in prediction of pathological response to neoadjuvant chemoradiotherapy and cancer-specific survival.

\section{Materials and methods}

\section{Patient Selection}

Patients with primary rectal adenocarcinoma were identified from the prospectively maintained Centre for Colorectal Disease database. Staging was performed in accordance with the American Joint Committee on Cancer Guidelines. ${ }^{22}$ Only patients with locally advanced rectal cancer who underwent neoadjuvant chemoradiotherapy and had subsequent rectal resection were included. Patients were excluded where a pre-treatment tumor biopsy was not available for review, if there were distant metastases at the time of diagnosis or if they had a cancer co-existing with ulcerative colitis, familial adenomatous polyposis or Lynch syndrome.

\section{Patient Management}

Pre-treatment tumor characteristics were determined with pelvic magnetic resonance imaging for $\mathrm{T}$ and $\mathrm{N}$ stage and computed tomography of the thorax, abdomen and pelvis for $\mathrm{M}$ stage. Radiotherapy was administered for a total dose of 45-50.4 Gray in 25-28 fractions of 1.8 Gray delivered over 6 weeks. 5-Fluorouracil was given concomitantly by protocol. ${ }^{26,27}$ Patients underwent surgical resection with curative intent $6-8$ weeks post completion of chemoradiotherapy.

\section{Histopathological Staging}

Pathological analysis using a standardized reporting template was performed on resected specimens and staged according to the American Joint Committee on Cancer. ${ }^{22} \mathrm{~A}$ tumor regression grade was assessed using a three-tier classification method. ${ }^{28,29}$ For the purposes of this study, pre-treatment rectal biopsies were retrospectively reviewed for evidence of intratumoral budding through a $\times 4$ lens, $(\times 40$ magnification) with confirmation of positive cases at $\times 10(\times 100$ magnification $)$ according to the method described by Giger et al, ${ }^{19}$ adapted from the Nakamura method of identifying peritumoral budding. ${ }^{30}$ Intra-tumoral budding was defined as a single cancer cell or a group of $<5$ detached tumor cells found in the stroma of the biopsy specimen. Thus, any budding seen at $\times 4$ and confirmed at $\times 10$ was deemed positive. An example of intra-tumoral budding is shown in Figure 1. Biopsies were reviewed independently by two pathologists (DG and $\mathrm{KS}$ ) blinded to the clinical and subsequent pathological outcomes, and in cases of discordance $(n=16)$, a consensus on budding status was obtained.

\section{Patient Follow-Up}

Follow-up was recorded through the prospectively maintained institutional colorectal cancer database.

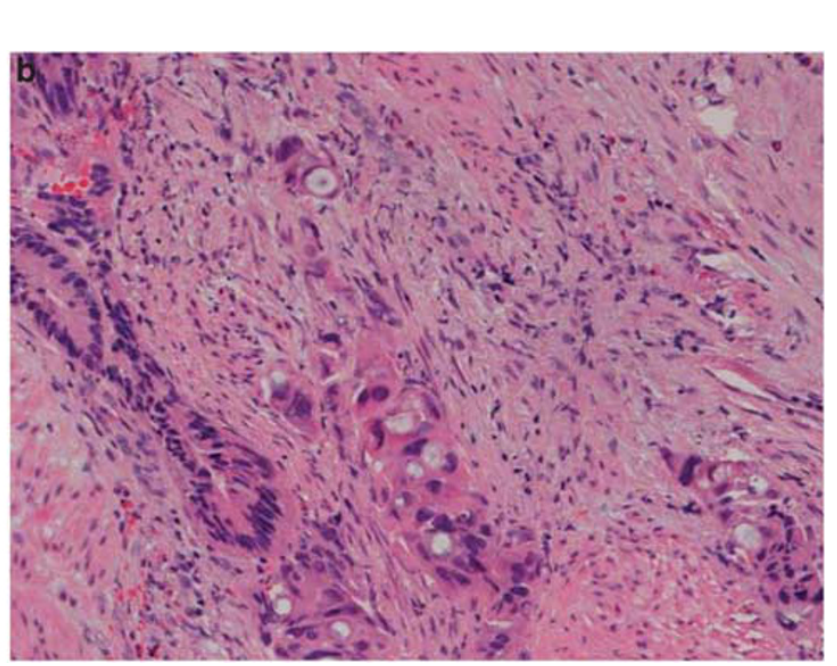

Figure 1 Biopsy samples of rectal adenocarcinoma stained with hematoxylin and eosin at $\times 200$ magnification. (a) Rectal cancer with no intra-tumoral budding. (b) Small groups and single tumor cells in a desmoplastic stroma typical of intra-tumoral budding. 
Primary care physicians were contacted as necessary to complete survival data.

\section{Statistical Analysis}

Results were analyzed using the Predictive Analytics Software (PASW 18.0.2, SPSS, Chicago, IL, USA). Comparative analyses of quantitative data were performed using the chi-squared test for categorical variables and Student's $t$-test for continuous variables. Spearman's rank test was used for correlation of budding with non-parametric variables. Tables were generated correlating budding status with tumor regression grade for determination of sensitivity and specificity. Univariate logistic regression was used to assess the effects of demographics and tumor characteristics on cancer-specific death, expressed as hazard ratios with 95\% confidence intervals (CIs). Variables with $P<0.200$ in univariate analysis were included in the multivariate logistic regression analysis. Kaplan-Meier curves were determined for analysis of 5-year survival based on budding status using the log rank test. All tests of significance were two-tailed, with $P<0.05$ indicating statistical significance.

\section{Results}

\section{Patient Demographics, Tumor and Treatment Characteristics}

Between 2003 and 2010, 185 patients underwent neoadjuvant chemoradiotherapy and subsequent resection for rectal cancer; 89 patients met the eligibility criteria. The median age was 62 years (range 30-84 years), and 64\% were male. Initial tumor staging with MR imaging (where available) identified 18\% (15/85) patients with American Joint Committee on Cancer Stage 2 cancer and the remaining had Stage 3 disease. Of the 38 patients who had MR re-imaging post neoadjuvant chemoradiotherapy, $20(53 \%)$ exhibited tumor downstaging. Twenty-five patients underwent abdominoperineal resection, 2 had ultra-low anterior resection with coloanal anastomosis, 19 underwent low anterior resection, 36 had standard anterior resection and the remainder either had pelvic exenteration or a Hartmann's procedure. Overall, $13 \%$ had a complete pathological response (tumor regression grade 1 ). In all, $50.2 \%$ had a partial response (tumor regression grade 2) while the remainder were graded as tumor regression grade 3 , denoting no response to chemoradiation. Median follow-up was 49 months (range 7-117 months).

\section{Budding in Pre-Treatment Samples}

Intra-tumoral budding was identified in the pretreatment tumor biopsy in 18 of the 89 patients $(20 \%)$. There were no differences in patient demographics or pre-treatment tumor characteristics between the two groups (Table 1).
Table 1 Comparison of patient demographics or pre-treatment tumor characteristics between those positive or negative for budding in the pre-treatment rectal cancer biopsy

\begin{tabular}{|c|c|c|c|}
\hline & \multicolumn{2}{|c|}{ Intra-tumoral budding } & \multirow[b]{2}{*}{$\mathrm{P}$-value } \\
\hline & $\begin{array}{l}\text { Budding } \\
(\mathrm{n}=18)\end{array}$ & $\begin{array}{l}\text { No budding } \\
\quad(\mathrm{n}=71)\end{array}$ & \\
\hline Mean age (years) & 59.3 (s.d. 8.6) & 62.3 (s.d. 12.8) & 0.224 \\
\hline Male sex (\%) & $12(67)$ & $45(63)$ & 0.795 \\
\hline Tumor site (\%) & & & 0.913 \\
\hline Low & $11(61)$ & $44(62)$ & \\
\hline Mid & $5(28)$ & $17(24)$ & \\
\hline High & $2(11)$ & $10(14)$ & \\
\hline T stage (\%) & & & 0.154 \\
\hline 2 & $0(0)$ & $8(12)$ & \\
\hline 3 & $15(88)$ & $54(81)$ & \\
\hline 4 & $2(13)$ & $5(8)$ & \\
\hline Node positive (\%) & $10(62)$ & $44(67)$ & 0.324 \\
\hline Operation type (\%) & & & 0.051 \\
\hline $\begin{array}{l}\text { Abdominoperineal } \\
\text { resection }\end{array}$ & 7 (39) & $18(25)$ & \\
\hline Low anterior resection & $4(22)$ & $17(24)$ & \\
\hline Anterior resection & $5(28)$ & $31(44)$ & \\
\hline Exenteration/other & $2(11)$ & $5(7)$ & \\
\hline
\end{tabular}

There were no significant differences between the two groups.

\section{Intra-Tumoral Budding Predicts Poor Pathological Response to Chemotherapy}

The presence of intra-tumoral budding in pretreatment biopsies was significantly associated with negative tumor prognostic factors found in the resected tumor specimen (Table 2). Intra-tumoral budding was strongly indicative of higher ypT stage (0.019), residual lymph node involvement $(P=0.018)$, poor differentiation $(P=0.005)$ and lymphovascular invasion $(P=0.004)$. There was a non-significant association with perineural invasion $(P=0.060)$ and higher tumor regression grade $(P=0.061)$. No patient with intra-tumoral budding in the pre-treatment biopsy had a complete pathological response to neoadjuvant chemoradiotherapy (tumor regression grade 1), giving a 100\% specificity and positive predictive value for non-response to neoadjuvant chemoradiotherapy (Table 3).

\section{Budding in Pre-Treatment Biopsy Predicts Poor Disease-Free Survival and Cancer-Specific Death}

The disease-free survival and cancer-specific death rate at 5 years were 72 and $18 \%$, respectively. In patients who developed recurrent disease and those who died, almost half of them exhibited budding in the pre-treatment rectal biopsy. Intratumoral budding was an independent predictor of increased local and distant recurrence, as well as cancer-specific death (Table 4 and Figure 2). Univariate and multivariate analysis of factors predisposing to cancer-specific death identified intratumoral budding as a significant predictor of cancer death (hazard ratio 3.51, 95\%CI 1.03-11.93, 
Table 2 Comparison of post neoadjuvant chemoradiotherapy tumor characteristics between those positive or negative for budding in the pre-treatment biopsy

\begin{tabular}{|c|c|c|c|}
\hline & \multicolumn{2}{|c|}{ Intra-tumoral budding } & \multirow[b]{2}{*}{$\mathrm{P}$-value } \\
\hline & $\begin{array}{l}\text { Budding } \\
(\mathrm{n}=18)\end{array}$ & $\begin{array}{l}\text { No budding } \\
(\mathrm{n}=71)\end{array}$ & \\
\hline T stage & & & $0.032^{\mathrm{a}}$ \\
\hline урТо & $0(0)$ & $10(14)$ & \\
\hline ypT1 & $0(0)$ & $6(8)$ & \\
\hline урТ2 & $4(22)$ & $16(23)$ & \\
\hline урТ3 & $11(61)$ & $33(46)$ & \\
\hline урT4 & $3(17)$ & $6(9)$ & \\
\hline Node positive & $11(61)$ & $22(31)$ & 0.018 \\
\hline \multicolumn{4}{|l|}{ Tumor differentiation ${ }^{\mathrm{b}}$} \\
\hline $\begin{array}{l}\text { Well/moderately } \\
\text { differentiated }\end{array}$ & $12(67)$ & $56(95)$ & 0.005 \\
\hline Poorly differentiated & $6(33)$ & $3(5)$ & 0.005 \\
\hline Lymphovascular invasion & $9(50)$ & 12 (18) & 0.004 \\
\hline Perineural invasion & $4(22)$ & $5(7)$ & 0.060 \\
\hline \multicolumn{4}{|l|}{ Tumor regression grade } \\
\hline Tumor regression grade 1 & $0(0)$ & $12(17)$ & 0.061 \\
\hline Tumor regression grade $2 / 3$ & $18(100)$ & $59(83)$ & 0.061 \\
\hline
\end{tabular}

Tumor budding was significantly associated with residual lymph node involvement, lymphovascular invasion and poorly differentiated tumors.

Values in parentheses are percentages.

${ }^{\text {a }}$ Spearman's rank test.

b These numbers exclude those 12 patients without budding who exhibited a tumor regression grade 1, as tumor differentiation is not applicable to these samples.

Table 3 Non-response to neoadjuvant chemoradiotherapy in those positive or negative for budding in the pre-treatment biopsy

\begin{tabular}{lccc}
\hline & \multicolumn{2}{c}{ Budding status } & \\
\cline { 2 - 3 } & Budding & No budding & Total \\
\hline Tumor regression grade & 0 & 12 & 12 \\
Tumor regression grade 1 & 18 & 59 & 77 \\
Tumor regression grade 2/3 & 18 & 71 & 89 \\
Total & &
\end{tabular}

Tumor budding has a sensitivity, specificity, positive predictive value and negative predictive value of $23.38,100,100$ and $16.90 \%$, respectively for determining non-response to neoadjuvant chemoradiotherapy.

$P=0.040$ ). The only tumor characteristic at surgical resection that also independently predicted death in multivariate analysis was tumor stage (Table 5). Tumor budding was not assessed in resected specimens.

\section{Discussion}

This study has found that the identification of budding in a pre-treatment biopsy of rectal cancer (intra-tumoral budding) is strongly predictive of a poor response to neoadjuvant chemoradiotherapy
Table 4 Relationship of intra-tumoral budding with long-term outcomes

\begin{tabular}{lcrr}
\hline & \multicolumn{2}{c}{ Intra-tumoral budding } & \\
\cline { 2 - 3 } & $\begin{array}{c}\text { Budding } \\
(\mathrm{n}=18)\end{array}$ & $\begin{array}{c}\text { No budding } \\
(\mathrm{n}=71)\end{array}$ & P-value \\
\hline Disease-free survival & $6(33)$ & $55(77)$ & $<0.001$ \\
Local recurrence & $6(33)$ & $7(10)$ & 0.012 \\
Distal recurrence & $8(44)$ & $11(15)$ & 0.007 \\
Cancer-specific death & $7(39)$ & $9(13)$ & 0.010 \\
\hline
\end{tabular}

Values in parentheses are percentages.

and is associated with poor long-term prognosis. This observation must now be considered in the context that at the time of diagnosis of locally advanced rectal cancer, clinical characteristics and tumor stage poorly predict tumor response to neoadjuvant chemoradiotherapy and thus overall prognosis. ${ }^{31,32}$ Other studies indicate that serological markers such as CEA are markers for tumor response to neoadjuvant chemoradiotherapy. ${ }^{23-25}$ Identification of patient subsets who are not likely to exhibit a complete pathological tumor response to neoadjuvant chemoradiotherapy must inform treatment decisions and help tailor individualized management. As there are no additional staining protocols required, intra-tumoral budding assessment can be performed at the time of pathological diagnosis at little or no extra cost.

This study was not designed to address the issue of reproducibility of budding in biopsy specimens. Apart from agreeing to use the criteria published by Giger et al, ${ }^{19}$ no training or validation sets were used. In this study, tumor budding status was assigned based on a consensus of two pathologist's interpretation. The study does not validate this method of identifying intra-tumoral budding compared with other methods but does identify two groups of patients with markedly different outcomes. Budding was denoted as either 'present' or 'absent' in the biopsy specimens-this is a practical solution, as biopsy samples often give a low yield of tumor material and often do not allow for assessment of multiple high-power fields. Interestingly, our positivity rate $(18 / 89,20 \%)$ was very similar to the $17 \%$ intra-tumoral budding rate reported by Giger et al. ${ }^{19}$ Although their study addressed reproducibility, only a small number of biopsy cases $(n=14)$ were subjected to intraobserver analysis.

Previous studies have correlated tumor budding in resected specimens with higher tumor regression grade. ${ }^{33,34}$ This study demonstrates that budding in the initial biopsy predicts residual adverse features post neoadjuvant chemoradiotherapy (higher ypT stage, positive lymph nodes, poorly differentiated tumors and lymphovascular invasion). Intra-tumoral budding was associated with tumor regression grade 

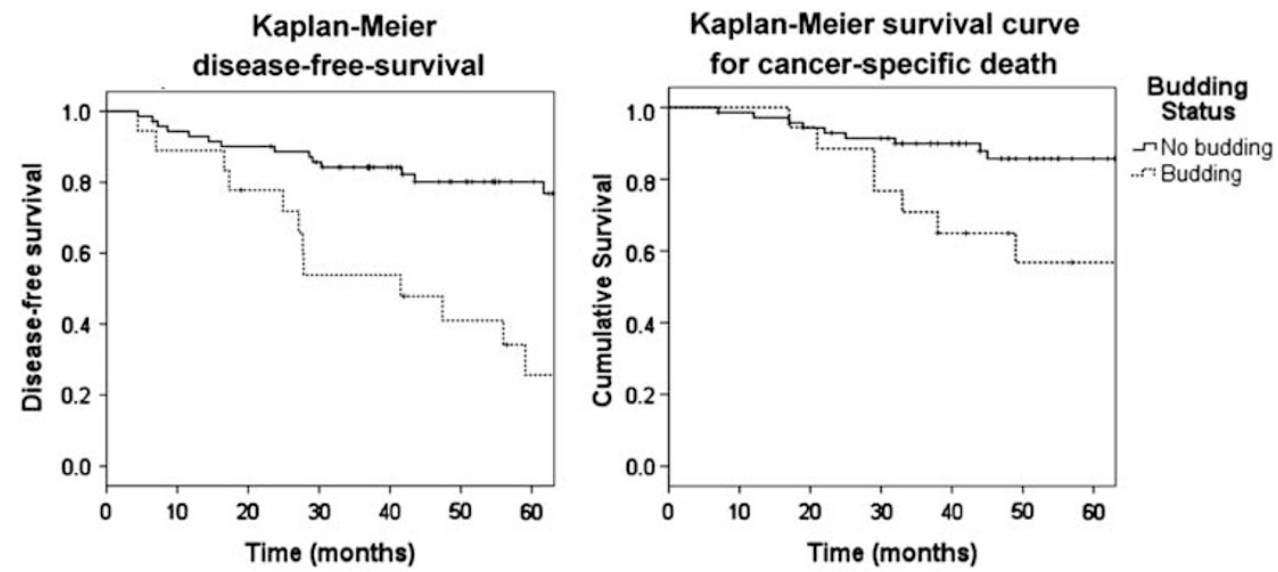

Figure 2 Kaplan-Meier survival curve relating to budding status in the pre-treatment biopsy. Patients with tumor budding in initial biopsy have a significantly lower 5 -year disease-free survival rate $(33.33 \mathrm{vs} 77.46 \%, P \leq 0.001)$ and lower cancer-specific survival rate (61.11 vs $87.32 \%, P=0.021)$.

Table 5 Univariate and multivariate logistic regression analysis of factors predisposing to cancer-specific death

\begin{tabular}{|c|c|c|c|c|}
\hline & \multicolumn{2}{|l|}{ Univariate analysis } & \multicolumn{2}{|l|}{ Multivariate analysis } \\
\hline & Hazard ratio (confidence interval) & $\mathrm{P}$-value & Hazard ratio (confidence interval) & $\mathrm{P}$-value \\
\hline \multicolumn{5}{|l|}{ Patient demographics } \\
\hline Age $\geq 75$ years & $1.34(0.39-4.98)$ & 0.606 & & \\
\hline Male sex & $2.07(0.61-6.98)$ & 0.235 & & \\
\hline Treated after 2006 & $0.851(0.30-2.46)$ & 0.765 & & \\
\hline \multicolumn{5}{|c|}{ Pre-treatment tumor characteristics } \\
\hline Budding & $3.88(1.22-12.38)$ & 0.017 & $3.51(1.03-11.93)$ & 0.040 \\
\hline Lower rectum & $0.64(0.22-1.85)$ & 0.403 & & \\
\hline Mid rectum & $1.91(0.61-5.97)$ & 0.261 & & \\
\hline Upper rectum & $0.83(0.16-4.18)$ & 0.818 & & \\
\hline Abdominoperineal resection & $1.08(0.34-3.47)$ & 0.893 & & \\
\hline Low anterior resection & $0.37(0.78-1.78)$ & 0.201 & & \\
\hline Anterior resection & $1.04(0.35-3.04)$ & 0.946 & & \\
\hline \multicolumn{5}{|l|}{ Pathological characteristics } \\
\hline Well differentiated & $1.24(0.23-6.57)$ & 0.802 & & \\
\hline Moderately differentiated & $0.86(0.28-2.61)$ & 0.791 & & \\
\hline Poorly differentiated & $4.12(0.97-17.45)$ & 0.041 & $1.42(0.25-8.05)$ & 0.690 \\
\hline урТ3/Т4 & $6.71(1.43-31.50)$ & 0.007 & $2.55(0.59-11.05)$ & 0.210 \\
\hline ypT4 only & $7.08(1.66-30.23)$ & 0.003 & $6.41(1.42-28.97)$ & 0.016 \\
\hline Node positive & $3.04(1.03-9.01)$ & 0.039 & $1.65(0.48-5.61)$ & 0.425 \\
\hline Lymphovascular invasion & $2.75(0.88-8.51)$ & 0.073 & $1.02(0.25-4.11)$ & 0.982 \\
\hline Perineural invasion & $4.06(0.96-17.20)$ & 0.044 & $1.93(0.37-10.18)$ & 0.440 \\
\hline Tumor regression grade 1 & $0.83(0.75-0.93)$ & 0.070 & $0.33(0.15-0.99)$ & 0.186 \\
\hline Tumor regression grade 2 & $1.19(0.41-3.43)$ & 0.748 & & \\
\hline Tumor regression grade 3 & $1.67(0.57-4.86)$ & 0.344 & & \\
\hline
\end{tabular}

ypT3/4 tumor category based on pathological analysis of the excised specimen following neoadjuvant chemoradiotherapy.

2 or 3 in $100 \%$ of cases. This suggests that tumors with budding at initial diagnosis are unlikely to exhibit a complete response to neoadjuvant chemoradiotherapy. However, only $25 \%$ of non-responsive cases (tumor regression grade 2 and 3) showed intratumoral budding; thus additional markers of nonresponse are required.

The present study found no differences in baseline clinical or tumor parameters between those biopsies with or without intra-tumoral budding. The poor prognostic outcomes in the former group suggest an aggressive phenotype, which may now be identified at initial biopsy. This group may be a target for escalation of therapy; however, it remains to be determined what course of action is most suited to these patients. Certainly they are a group in whom further study is required to improve outcomes.

The results are limited by the retrospective nature of database studies. Type II errors (such as the 
apparent lack of correlation of tumor budding with perineural invasion) may have occurred due to the sample size. Use of intra-tumoral budding as a prognostic marker will be limited by its diagnostic reproducibility. A recent study clarified some issues surrounding this; ${ }^{14}$ reproducibility of this currently described method is a significant issue and will need to be demonstrated in future studies.

Intra-tumoral budding is a marker of poor prognosis and poor response to neoadjuvant chemoradiotherapy. If confirmed in other series, intratumoral budding may be an indication to avoid neoadjuvant chemoradiotherapy in its present form.

\section{Disclosure/conflict of interest}

The authors declare no conflict of interest.

\section{References}

1 Morodomi T, Isomoto H, Shirouzu K, et al. An index for estimating the probability of lymph node metastasis in rectal cancers. Lymph node metastasis and the histopathology of actively invasive regions of cancer. Cancer 1989;63:539-543.

2 Ueno H, Murphy J, Jass JR, et al. Tumour 'budding' as an index to estimate the potential of aggressiveness in rectal cancer. Histopathology 2002;40:127-132.

3 O’Brien CA, Pollett A, Gallinger S, et al. A human colon cancer cell capable of initiating tumour growth in immunodeficient mice. Nature 2007;445:106-110.

4 Prall F. Tumour budding in colorectal carcinoma. Histopathology 2007;50:151-162.

5 Kanazawa H, Mitomi H, Nishiyama Y, et al. Tumour budding at invasive margins and outcome in colorectal cancer. Colorectal Dis 2008;10:41-47.

6 Kazama S, Watanabe T, Ajioka Y, et al. Tumour budding at the deepest invasive margin correlates with lymph node metastasis in submucosal colorectal cancer detected by anticytokeratin antibody CAM5.2. Br J Cancer 2006;94:293-298.

7 Glasgow SC, Bleier JI, Burgart LJ, et al. Meta-analysis of histopathological features of primary colorectal cancers that predict lymph node metastases. J Gastrointest Surg 2012;16:1019-1028.

8 Kevans D, Wang LM, Sheahan K, et al. Epithelialmesenchymal transition (EMT) protein expression in a cohort of stage II colorectal cancer patients with characterized tumor budding and mismatch repair protein status. Int J Surg Pathol 2011;19:751-760.

9 Guzinska-Ustymowicz K. The role of tumour budding at the front of invasion and recurrence of rectal carcinoma. Anticancer Res 2005;25:1269-1272.

10 Prall F, Nizze H, Barten M. Tumour budding as prognostic factor in stage I/II colorectal carcinoma. Histopathology 2005;47:17-24.

11 Wang LM, Kevans D, Mulcahy H, et al. Tumor budding is a strong and reproducible prognostic marker in T3No colorectal cancer. Am J Surg Pathol 2009;33: 134-141.

12 Suzuki A, Togashi K, Nokubi M, et al. Evaluation of venous invasion by Elastica van Gieson stain and tumor budding predicts local and distant metastases in patients with T1 stage colorectal cancer. Am J Surg Pathol 2009;33:1601-1607.

13 Mitrovic B, Schaeffer DF, Riddell RH, et al. Tumor budding in colorectal carcinoma: time to take notice. Mod Pathol 2012;25:1315-1325.

14 Puppa G, Senore C, Sheahan K, et al. Diagnostic reproducibility of tumour budding in colorectal cancer: a multicentre, multinational study using virtual microscopy. Histopathology 2012;61:562-575.

15 Karamitopoulou E, Zlobec I, Kolzer V, et al. Proposal for a 10-high-power-fields scoring method for the assessment of tumor budding in colorectal cancer. Mod Pathol 2013;26:295-301.

16 Horcic M, Koelzer VH, Karamitopoulou E, et al. Tumor budding score based on 10 high-power fields is a promising basis for a standardized prognostic scoring system in stage II colorectal cancer. Hum Pathol 2013;44:697-705.

17 Morodomi T. [Clinicopathological studies of advanced rectal cancers-prediction of the degree of lymph node metastasis from histopathological finding of preoperative biopsy specimens]. Nihon Geka Gakkai Zasshi 1988;89:352-364.

18 Lugli A, Vlajnic T, Giger O, et al. Intratumoral budding as a potential parameter of tumor progression in mismatch repair-proficient and mismatch repair-deficient colorectal cancer patients. Hum Pathol 2011;42:1833-1840.

19 Giger OT, Comtesse SC, Lugli A, et al. Intra-tumoral budding in preoperative biopsy specimens predicts lymph node and distant metastasis in patients with colorectal cancer. Mod Pathol 2012;25:1048-1053.

20 Merkel S, Mansmann U, Papadopoulos T, et al. The prognostic inhomogeneity of colorectal carcinomas Stage III: a proposal for subdivision of Stage III. Cancer 2001;92:2754-2759.

21 Nagtegaal ID, Gosens MJ, Marijnen CA, et al. Combinations of tumor and treatment parameters are more discriminative for prognosis than the present TNM system in rectal cancer. J Clin Oncol 2007;25:1647-1650.

22 Edge SB, Compton CC. The American Joint Committee on Cancer: the 7th edition of the AJCC cancer staging manual and the future of TNM. Ann Surg Oncol 2010;17:1471-1474.

23 Moreno Garcia V, Cejas P, Blanco Codesido M, et al. Prognostic value of carcinoembryonic antigen level in rectal cancer treated with neoadjuvant chemoradiotherapy. Int J Colorectal Dis 2009;24:741-748.

24 Das P, Skibber JM, Rodriguez-Bigas MA, et al. Predictors of tumor response and downstaging in patients who receive preoperative chemoradiation for rectal cancer. Cancer 2007;109:1750-1755.

25 Park YA, Sohn SK, Seong J, et al. Serum CEA as a predictor for the response to preoperative chemoradiation in rectal cancer. J Surg Oncol 2006;93:145-150.

26 Royal College of Surgeons of England and Association of Coloproctology of Great Britain and Ireland Guidelines for the Management of Colorectal Cancer, 3rd edn. Royal College of Surgeons: London, UK, 2007.

27 Tjandra JJ, Kilkenny JW, Buie WD, et al. Practice parameters for the management of rectal cancer (revised). Dis Colon Rectum 2005;48:411-423.

28 Ryan R, Gibbons D, Hyland JM, et al. Pathological response following long-course neoadjuvant chemoradiotherapy for locally advanced rectal cancer. Histopathology 2005;47:141-146.

29 Beddy D, Hyland JM, Winter DC, et al. A simplified tumor regression grade correlates with survival in 
locally advanced rectal carcinoma treated with neoadjuvant chemoradiotherapy. Ann Surg Oncol 2008; 15:3471-3477.

30 Nakamura T, Mitomi H, Kikuchi S, et al. Evaluation of the usefulness of tumor budding on the prediction of metastasis to the lung and liver after curative excision of colorectal cancer. Hepatogastroenterology 2005; 52:1432-1435.

31 Quah HM, Chou JF, Gonen M, et al. Pathologic stage is most prognostic of disease-free survival in locally advanced rectal cancer patients after preoperative chemoradiation. Cancer 2008;113:57-64.
32 Puppa G, Sonzogni A, Colombari R, et al. TNM staging system of colorectal carcinoma: a critical appraisal of challenging issues. Arch Pathol Lab Med 2010;134: 837-852.

33 Huebner M, Wolff BG, Smyrk TC, et al. Partial pathologic response and nodal status as most significant prognostic factors for advanced rectal cancer treated with preoperative chemoradiotherapy. World J Surg 2012;36:675-683.

34 Du C, Xue W, Li J, et al. Morphology and prognostic value of tumor budding in rectal cancer after neoadjuvant radiotherapy. Hum Pathol 2012;43:1061-1067. 